\title{
The Mechanics of Undulatory Locomotion: The Mixed Kinematic and Dynamic Case
}

\author{
Jim Ostrowski Joel Burdick Andrew D. Lewis Richard M. Murray \\ Division of Engineering and Applied Science \\ California Institute of Technology, Pasadena, CA 91125
}

\begin{abstract}
This paper studies the mechanics of undulatory locomotion. This type of locomotion is generated by a coupling of internal shape changes to external nonholonomic constraints. Employing methods from geometric mechanics, we use the dynamic symmetries and kinematic constraints to develop a specialized form of the $d y$ namic equations which govern undulatory systems. These equations are written in terms of physically meaningful and intuitively appealing variables that show the role of internal shape changes in driving locomotion.
\end{abstract}

\section{Introduction}

A significant body of research has been developed in the area of robotic locomotion. However, prior studies have been focused either on a particular set of assumptions or a particular robot morphology. For example, numerous investigators have studied and demonstrated quasi-static, multi-legged locomotion [16]. Beginning with Raibert [14], hopping robots have received considerable attention [9], [2]. Bipedal walking and running has also been an active area of study [12], [6]. Other researchers have considered and implemented various forms of "snake-like" locomotion schemes [5], [4] and investigated the geometry of amoeba swimming through a viscous fluid [15]. However, to date there exists no unifying methodology for analyzing or controlling robot locomotion. Ultimately, we seek a mechanics theory and a control theory for robotic locomotion which is uniformly applicable to a broad class of locomotory problems. This paper introduces a unifying mechanics principle for undulatory locomotion.

Definition: Undulatory locomotion is the process of generating net displacements of a robotic mechanism via a coupling of internal deformations to an interaction between the robot and its environment.

Common biological examples of undulatory locomotion include worms, snakes, amoeba, and fish. In this paper, we limit these interactions to those modeled by nonholonomic kinematic constraints. This restriction allows us to model a rich class of systems and results in enough structure to make the problem tractable. We believe that the framework presented here will provide the basis for an undulatory locomotion control theory, and will ultimately be extended to a very large class of locomotory systems.

This work has several goals and contributions. First we show that locomotion problems can naturally be cast in the framework of principal fiber bundles. Second, using this structure, we derive a specialized form of the dynamical equations for mechanical systems with Lagrangian symmetries and nonholonomic constraints (which are characteristic of many undulatory locomotors). Third, we show how these results lead to a simple and appealing insight into undulatory locomotion. Finally, we show that the framework presented here is in fact a superset of prior work on the mechanics of wheeled nonholonomic vehicles and free-floating satellites.

The results in this paper draw significantly from recent results in Lagrangian mechanics due to Koiller [10] and Bloch et al., [3]. We also note that the role of connections in problems of locomotion has been explored in [7], where results were developed regarding mechanics and control in the case of purely kinematic constraints.

\section{Mechanics}

It is always possible to divide a locomoting robot's configuration variables into two classes. The first class of variables describes the position of the robot. We define this to be the displacement of a coordinate frame attached to the moving robot mechanism with respect to a fixed reference frame. Since robots move in Euclidean space, the set of body frame displacements is $S E(n), n \leq 3$, or one of its subgroups-i.e., a Lie group. The second class of variables defines the internal configuration, or shape, of the mechanism. We require only that the set of all possible shapes be described by a manifold, $M$. Hence, the Lie group, $G$, together with the shape space, $M$, form the total configuration space of the system, which we denote by $Q=G \times M$. A given configuration is denoted by $q \in Q$.

Since we are working with mechanical systems, we will assume the existence of a Lagrangian function, $L(q, \dot{q})$, on $T Q$, the tangent bundle of $Q$. In the absence of constraints, the robot's dynamical equations can be derived from Lagrange's equations:

$$
\frac{d}{d t}\left(\frac{\partial L}{\partial \dot{q}^{i}}\right)-\frac{\partial L}{\partial q^{i}}-\tau_{i}=0,
$$

where $\tau$ is a forcing function. In general, though, undulatory locomotion requires some type of interaction with the environment, which we will model as a constraint. These constraints can take many forms, including viscous friction, no-slip wheel conditions, and interaction of a surface with a viscous fluid or air. Let us restrict our attention to constraints which are linear in the velocities. Given 

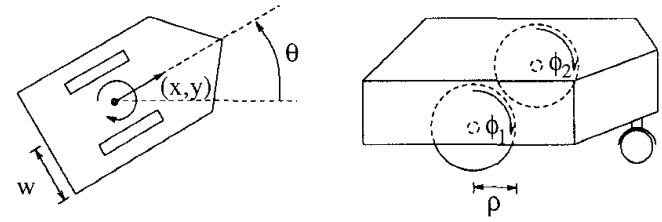

Fig. 1. Two wheeled planar mobile robot.

$k$ such constraints, we can write them as a vector-valued set of $k$ equations:

$$
\omega_{j}^{i}(q) \dot{q}^{j}=0, \quad \text { for } \quad i=1 \ldots k .
$$

This class of constraints includes most commonly investigated nonholonomic constraints.

The constraints can be incorporated into the dynamics through the use of Lagrange multipliers. That is, Eq. 1 is modified by adding a force of constraint with an unknown multiplier, $\lambda$.

$$
\frac{d}{d t}\left(\frac{\partial L}{\partial \dot{q}^{i}}\right)-\frac{\partial L}{\partial q^{i}}+\lambda_{j} \omega_{i}^{j}-\tau_{i}=0
$$

Example 1. Consider the two wheeled planar mobile robot shown in Figure 1. The robot's position, $(x, y, \theta) \in$ $S E(2)$, is measured via a frame located at the center of the wheel base. The position of the wheels is measured relative to vertical and is denoted $\left(\phi_{1}, \phi_{2}\right)$. Each wheel is assumed to rotate independently and without slipping. The configuration space is then $Q=G \times M=S E(2) \times$ $\left(\mathbb{S}^{1} \times \mathbb{S}^{1}\right)$. The Lagrangian for this problem is

$$
L=\frac{1}{2} m\left(\dot{x}^{2}+\dot{y}^{2}\right)+\frac{1}{2} J \dot{\theta}^{2}+\frac{1}{2} J_{w}\left(\dot{\phi}_{1}^{2}+\dot{\phi}_{2}^{2}\right),
$$

where $m$ is the mass of the robot, $J$ is its inertia, and $J_{w}$ is the inertia of each of the wheels. The constraints defining the no-slip condition can be written as in Eq. 2:

$$
\begin{aligned}
\dot{x} \cos \theta+\dot{y} \sin \theta-\frac{\rho}{2}\left(\dot{\phi}_{1}+\dot{\phi}_{2}\right) & =0 \\
-\dot{x} \sin \theta+\dot{y} \cos \theta & =0 \\
\dot{\theta}-\frac{\rho}{2 w}\left(\dot{\phi}_{1}-\dot{\phi}_{2}\right) & =0 .
\end{aligned}
$$

The equations of motion can then be derived using Eq. 3 . In this case, however, the kinematic constraints provide an immediate way of determining the robot's motion as a function of internal shape changes since there are three constraints on the three dimensional group of body displacements. If we make the standard assumption that the base variables are controllable, then given the time evolution of $\phi_{1}$ and $\phi_{2}$, we can completely solve for the motion of the robot using $\mathrm{Eq}$. 4, which we rewrite in a slightly more revealing manner:

$$
\left(\begin{array}{ccc}
\cos \theta & \sin \theta & 0 \\
-\sin \theta & \cos \theta & 0 \\
0 & 0 & 1
\end{array}\right)\left(\begin{array}{l}
\dot{x} \\
\dot{y} \\
\dot{\theta}
\end{array}\right)=\left(\begin{array}{c}
\frac{\rho}{2}\left(\dot{\phi}_{1}+\dot{\phi}_{2}\right) \\
0 \\
\frac{\rho}{2 w}\left(\dot{\phi}_{1}-\dot{\phi}_{2}\right)
\end{array}\right) .
$$

That is, the motion in the group variables, $(\dot{x}, \dot{y}, \dot{\theta})$, is strictly a function of the internal shape velocities $\left(\dot{\phi}_{1}, \dot{\phi}_{2}\right)$.

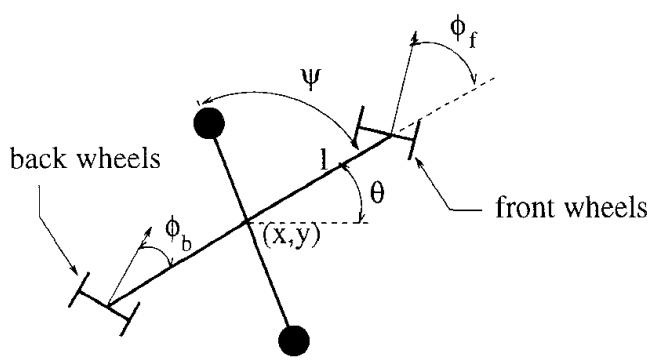

Fig. 2. The simplified model of the Snakeboard.

Example 2. Next we turn to an example which will be used throughout the paper for the purposes of illustration. The simplified model of the Snakeboard (c.f., [11]) is shown in Figure 2 and consists of a rigid body connecting two sets of wheels whose rotations can be independently specified. Attached is a momentum wheel which rotates about the center of mass, thereby exerting a torque on the lower portion of the board. The snakeboard's position variables are $(x, y, \theta) \in G=S E(2)$, and are determined by a frame affixed to its center of mass. The internal shape variables are $\left(\psi, \phi_{b}, \phi_{f}\right)$, and so the base space is $\mathbb{S}^{1} \times \mathbb{S}^{1} \times \mathbb{S}^{1}=M$. The configuration space is $Q=G \times M=S E(2) \times \mathbb{S}^{1} \times \mathbb{S}^{1} \times \mathbb{S}^{1}$. The Lagrangian is

$$
\begin{aligned}
L & =\frac{1}{2} m\left(\dot{x}^{2}+\dot{y}^{2}\right)+\frac{1}{2} J \dot{\theta}^{2}+\frac{1}{2} J_{r}(\dot{\psi}+\dot{\theta})^{2} \\
& +\frac{1}{2} J_{w}\left(\left(\dot{\phi}_{b}+\dot{\theta}\right)^{2}+\left(\dot{\phi}_{f}+\dot{\theta}\right)^{2}\right) .
\end{aligned}
$$

Control torques at the rotor and wheels are assumed, so

$$
\tau=\left(0,0,0, \tau_{\psi}, \tau_{b}, \tau_{f}\right) .
$$

The assumption that the wheels do not slip in the direction of the wheel axes determines two constraints that can be written as linear functions of the velocities:

$$
\begin{aligned}
& -\sin \left(\phi_{f}+\theta\right) \dot{x}+\cos \left(\phi_{f}+\theta\right) \dot{y}+l \cos \left(\phi_{f}\right) \dot{\theta}=0 \\
& -\sin \left(\phi_{b}+\theta\right) \dot{x}+\cos \left(\phi_{b}+\theta\right) \dot{y}-l \cos \left(\phi_{b}\right) \dot{\theta}=0 .
\end{aligned}
$$

Notice that for the snakeboard we no longer have enough kinematic constraints to uniquely define the motion of the robot. For this reason, the simple technique of using the constraints to solve for the robot's motion as a function of shape changes that was employed in the first example is no longer viable. The snakeboard's dynamics must come into play. Thus, we are relegated to using Eqs. 2 and 3 to define the robot's dynamics and explicitly solving for $k$ unknown Lagrange multipliers. There are a number of drawbacks to this approach. First, the system is equivalent to $2 n+k$ first order differential equations. Second, physical intuition is often lost when eliminating the Lagrange multipliers. That is, we do not have a relationship, such as Eq. 5, in which the effect of internal shape changes on robot motion is readily apparent. Third, it is difficult to incorporate into Eq. 3 any special features which might simplify the ensuing analysis. With 
these issues in mind, we now embark upon an alternate approach in order to make use of the additional structure given to us by the inherent symmetries found in problems of locomotion.

\section{Mechanics with symmetries}

\subsection{Mathematical background}

We begin by introducing some general mathematical concepts, which will be illustrated by the snakeboard example. First, we show that locomotion systems can be modeled on a principal fiber bundle and review some ideas that are associated with them.

Recall the division of the configuration space, $Q=$ $G \times M$. Such a configuration space is termed a trivial fiber bundle. $G$ is called the fiber, and $Q$ is said to be fibered over the base space, $M . Q$ is "trivial" because the product structure is global. In our context, the words fiber and base are interchangeable with robot position and internal shape, respectively. There are two natural projections which we will use. Given a point $(g, r) \in G \times M=Q$, define these projections as $\pi_{1}: Q \rightarrow G:(g, r) \mapsto g$ and $\pi_{2}: Q \rightarrow M:(g, r) \mapsto r$.

The use of a Lie group will be important for describing the robot's motion through its environment. Formally, the displacement of the robot's body fixed frame is considered as a left translation. That is, if the robot's initial position is denoted by $g$, and it is displaced by an amount $h$, then its final position is $h g$. This displacement can be thought of as a map $L_{h}: G \rightarrow G$ given by $L_{h}(g)=h g$ for $g \in G$. The left translation induces a left action of $G$ on $Q$.

Definition 3. A left action of a Lie group $G$ on a manifold $Q$ is a smooth map $\Phi: G \times Q \rightarrow Q$ such that: (1) $\Phi(e, q)=q$ for all $q \in Q$, and $e$ the identity element of $G$; and $(2) \Phi(h, \Phi(g, q))=\Phi(h g, q)$ for every $g, h \in G$ and $q \in Q$.

It will be useful to consider the left action as a map from $Q$ into $Q$, with the element $h \in G$ held fixed. Notationally, $\Phi_{h}: Q \rightarrow Q$ is given by $(g, r) \mapsto(\Phi(h, g), r)=$ $(h g, r)$. The lifted action, which describes the effect of $\Phi_{h}$ on velocity vectors in $T Q$, is the tangent map of $\Phi_{h}$. This is the linear map, $D_{q} \Phi_{h}: T_{q} Q \rightarrow T_{h q} Q$ (often denoted $\left.T_{q} \Phi_{h}\right)$.

Definition 4. Let $M$ be a manifold and $G$ a Lie group. A trivial principal fiber bundle with base $M$ and structure group $G$ consists of the manifold $Q=G \times M$ together with the free left action of $G$ on $Q$ given by left translation on the group variable: $\Phi_{h}(g, r)=(h g, r)$ for $r \in M$ and $h, g \in G$.

That is, our bundle configuration space has additional structure arising from the Lie group component. This additional structure is important for the ensuing developments.
Example 2: (cont'd) The configuration space for the snakeboard is $Q=G \times M=S E(2) \times\left(\mathbb{S}^{1} \times \mathbb{S}^{1} \times \mathbb{S}^{1}\right)$, and a configuration is denoted $q=\left(x, y, \theta, \psi, \phi_{b}, \phi_{f}\right)$. The action is the left action of $S E(2)$ on itself. Given $h=$ $\left(a_{1}, a_{2}, \alpha\right) \in S E(2)$,

$$
\begin{gathered}
\Phi_{h}(q)=\left(x \cos \alpha-y \sin \alpha+a_{1}, x \sin \alpha+y \cos \alpha+a_{2},\right. \\
\left.\theta+\alpha, \psi, \phi_{b}, \phi_{f}\right) .
\end{gathered}
$$

From this the lifted action is easily computed as

$$
\begin{gathered}
D_{q} \Phi_{h}\left(v_{q}\right)=\left(v_{x} \cos \alpha-v_{y} \sin \alpha, v_{x} \sin \alpha+v_{y} \cos \alpha\right. \\
\left.v_{\theta}, v_{\psi}, v_{b}, v_{f}\right),
\end{gathered}
$$

where $v_{q}=\left(v_{x}, v_{y}, v_{\theta}, v_{\psi}, v_{b}, v_{f}\right) \in T_{q} Q$ is a point in the tangent space of $Q$ at $q$.

Associated with a Lie group, $G$, is its Lie algebra, denoted $\mathfrak{g}$. The Lie algebra can be identified with $T_{e} G$ and generates $G$ via the exponential mapping, exp : $\mathfrak{g} \rightarrow G$ (see [1]). The exponential mapping also associates with each $\xi \in \mathfrak{g}$ a vector field on $G$, and by extension on $Q=G \times M$, called the infinitesimal generator, $\xi_{Q}$, given by

$$
\xi_{Q}(q)=\left.\frac{d}{d s}(\Phi(\exp (s \xi), q))\right|_{s=0} .
$$

Each infinitesimal generator is tangent to the fiber, and the set of all such vectors at $q \in Q$ forms a subspace of $T_{q} Q$ called the vertical subspace,

$$
V_{q} Q=\left\{\xi_{Q}(q) \in T_{q} Q \mid \xi \in \mathfrak{g}\right\} .
$$

Eq. 8 takes elements of the Lie algebra and maps them to infinitesimal generators. It is an isomorphism between $\mathfrak{g}$ and $V_{q} Q$. This implies that any vector $v_{q} \in V_{q} Q$ can be written as the infinitesimal generator at $q$ of some Lie algebra element, i.e., $v_{q}=\xi_{Q}(q)$ for some $\xi \in \mathfrak{g}$.

Example 2: (cont'd) The Lie algebra for $S E(2)$ is denoted se(2), and the relationship between an element, $\xi=\left(a_{1}, a_{2}, \alpha\right) \in s e(2)$, and the corresponding infinitesimal generator on $T Q$ is given by

$$
\xi_{Q}\left(x, y, \theta, \psi, \phi_{b}, \phi_{f}\right)=\left(a_{1}-y \alpha, a_{2}+x \alpha, \alpha, 0,0,0\right) .
$$

The vertical subspace is given trivially by $T G \times\{0\}$

$$
\begin{aligned}
V_{q} Q & =\left\{\left(v_{q}, w_{q}\right) \in T_{\pi_{1}(q)} G \times T_{\pi_{2}(q)} M=T_{q} Q \mid w_{q}=0\right\} \\
& =\operatorname{sp}\left\{\frac{\partial}{\partial x}, \frac{\partial}{\partial y}, \frac{\partial}{\partial \theta}\right\} .
\end{aligned}
$$

\subsection{Noether's theorem}

Conservation laws (e.g., conserved linear and angular momentum) naturally arise when a Lagrangian remains invariant under the action of a Lie group, as stated in Noether's theorem [1], [3]:

Theorem 5. (Noether) Let $L$ be a Lagrangian which is invariant under the action of a Lie group, $G$, (i.e., $\left.L\left(\Phi_{h}(q), D_{q} \Phi_{h} v_{q}\right)=L\left(q, v_{q}\right) \forall h \in G, v_{q} \in T_{q} Q\right)$. Then, for all curves, $c(t):[a, b] \rightarrow Q$ satisfying Lagrange's equations (Eq. 1), we have that

$$
\frac{d}{d t}\left\langle\frac{\partial L}{\partial \dot{q}}(\dot{c}(t)) ; \xi_{Q}(c(t))\right\rangle=0
$$


for all $\xi \in \mathfrak{g}$. Equivalently, $\dot{p}=0$, where $p=\left\langle\frac{\partial L}{\partial \dot{q}} ; \xi_{Q}\right\rangle$ is the generalized momentum.

For the case in which $G$ is $S E(2)$ or $S E(3)$, Noether's theorem is equivalent to conservation of linear and angular momentum. However, undulatory locomotion relies on some type of interaction with the environment. Unfortunately, conservation laws are not necessarily preserved in the presence of the constraints which are inherent to undulatory locomotion. The next section describes an extension to the classical theory that combines the effects of symmetries and constraints.

\subsection{Symmetries with constraints}

Given the constraints as in Eq. 2, we can write the constraint distribution (i.e., the set of all velocities that satisfy the constraints) as

$$
\mathcal{D}_{q}=\left\{v_{q} \in T_{q} Q \mid \omega_{j}^{i} v_{q}^{j}=0, \forall i=1, \ldots, k\right\} .
$$

The constraints are said to act vertically if the constrained fiber distribution,

$$
\mathcal{S}=\mathcal{D} \cap V Q
$$

is nonempty. Assuming this to be true, we have the following proposition, first developed in [3]. For proofs of this proposition and those to follow, the reader is referred to [3], [13].

Proposition 6. Let $L$ and $\mathcal{D}$ define a constrained system on $Q=G \times M$ whose Lagrangian is $G$-invariant. If $c$ is a curve which satisfies the Lagrange-d'Alembert equations (Eq. 3) for a system with nonholonomic constraints (Eq. 2), then the following generalized momentum equation holds for all vector fields, $\xi_{Q}^{c} \in \mathcal{S}$ :

$$
\frac{d}{d t} p^{c}=\frac{\partial L}{\partial \dot{q}^{i}}\left(\frac{d}{d t}\left[\xi^{c}(c(t))\right]\right)_{Q}^{i}+\tau_{i}\left(\xi^{c}(c(t))\right)_{Q}^{i}
$$

where

$$
p^{c}=\frac{\partial L}{\partial \dot{q}^{i}}\left(\xi^{c}(c(t))_{Q}^{i}\right.
$$

is the constrained momentum.

That is, in the presence of constraints, momentum-like quantities exist, but they may not be conserved. Eq. 12 determines how the momentum-like quantity, $p^{c}$, evolves. The non-conservation of momentum-like quantities is the key to dynamic undulatory locomotion. It describes why the snakeboard can start from rest and build up momentum, even though no external forces act on the system [11].

Example 2: (cont'd) An easy calculation shows that the snakeboard Lagrangian, Eq. 6 , is invariant with respect to the $S E(2)$ group action. The wheel constraints of Eq. 7 can be expressed as a constraint distribution:

$$
\mathcal{D}_{q}=\operatorname{sp}\left\{a \frac{\partial}{\partial x}+b \frac{\partial}{\partial y}+c \frac{\partial}{\partial \theta}, \frac{\partial}{\partial \psi}, \frac{\partial}{\partial \phi_{b}}, \frac{\partial}{\partial \phi_{f}}\right\},
$$

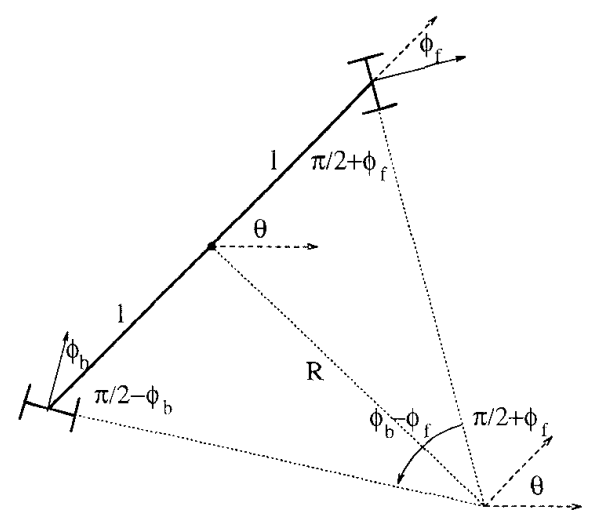

Fig. 3. Instantaneous center of rotation

where

$$
\begin{aligned}
a & =-l\left[\cos \phi_{b} \cos \left(\phi_{f}+\theta\right)+\cos \phi_{f} \cos \left(\phi_{b}+\theta\right)\right] \\
b & =-l\left[\cos \phi_{b} \sin \left(\phi_{f}+\theta\right)+\cos \phi_{f} \sin \left(\phi_{b}+\theta\right)\right] \\
c & =\sin \left(\phi_{b}-\phi_{f}\right) .
\end{aligned}
$$

The vertical distribution was defined in Eq. 9, and so the constrained fiber distribution is:

$$
\mathcal{S}_{q}=\mathcal{D}_{q} \cap V_{q} Q=\operatorname{span}\left\{a \frac{\partial}{\partial x}+b \frac{\partial}{\partial y}+c \frac{\partial}{\partial \theta}\right\} .
$$

Note, the constrained fiber distribution physically corresponds to instantaneous rotations of the snakeboard about a point where the two snakeboard wheel axes intersect (Figure 3).

This basis for $\mathcal{S}$ satisfies the necessary conditions given in Proposition 6. For the snakeboard, the constrained momentum of Eq. 13 is computed as

$$
\begin{aligned}
p^{c} & =\left\langle\frac{\partial L}{\partial \dot{q}} ;\left(\xi^{c}\right)_{Q}(q)\right\rangle \\
& =\left\langle\left(m \dot{x}, m \dot{y}, \hat{J} \dot{\theta}, J_{r} \dot{\psi}, J_{w} \dot{\phi}_{b}, J_{w} \dot{\phi}_{f}\right) ;(a, b, c, 0,0,0)\right\rangle \\
& =m a \dot{x}+m b \dot{y}+\hat{J} c \dot{\theta}+J_{r} c \dot{\psi}+J_{w} c\left(\dot{\phi}_{b}+\dot{\phi}_{f}\right) \\
& =\left(m R^{2}+\hat{J}_{c}\right) \dot{\theta}+J_{r} c \dot{\psi}+J_{w} c\left(\dot{\phi}_{b}+\dot{\phi}_{f}\right),
\end{aligned}
$$

where $\xi_{Q}^{c} \in \mathcal{S}, \hat{J}=J+J_{r}+2 J_{w}$ is the sum of the moments of inertia, and $R$ is the radius from the instantaneous center of rotation to the snakeboard's center of mass (Fig. 3). Thus, $p^{c}$ corresponds to the snakeboard's angular momentum about the instantaneous center of rotation. If the front and back wheels were fixed, this momentum would be conserved, as the wheels would provide a holonomic constraint forcing the snakeboard to rotate about the fixed center. For the snakeboard, the constrained momentum is one-dimensional. In general we would derive momenta corresponding to each unconstrained degree of freedom along the group orbit, i.e., the number of momenta would equal $\operatorname{dim} \mathcal{S}$. The generalized momentum equation, Eq. 12, for the snakeboard is:

$$
\dot{p}^{c}=m \dot{a} \dot{x}+m \dot{b} \dot{y}+\hat{J} \dot{c} \theta+J_{r} \dot{c} \dot{\psi}+J_{w} \dot{c}\left(\dot{\phi}_{b}+\dot{\phi}_{f}\right)
$$




\subsection{Constructing the connection}

We now introduce a key concept in the theory of principal fiber bundles which also has an important role in locomotion analysis.

Definition 7. [8] A connection is an assignment of a horizontal subspace, $H_{q} Q \subset T_{q} Q$, for each point $q \in Q$ such that

(1) $T_{q} Q=V_{q} Q \oplus H_{q} Q$,

(2) $D_{q} \Phi_{h} H_{q} Q=H_{h \cdot q} Q$, for every $q \in Q$ and $h \in G$, and

(3) $H_{q} Q$ depends smoothly on $q$.

Condition (1) implies that $T_{q} Q$ can everywhere be divided into a vertical subspace, $V_{q} Q$, and a horizontal subspace, $H_{q} Q$. Connections are useful because of the following fact. The horizontal subspace defined by the connection is everywhere isomorphic to the tangent space of the base: $H_{q} Q \simeq T_{\pi_{2}(q)} M$. The horizontal lift is the isomorphism which maps vectors in $T_{\pi_{2}(q)} M$ to the corresponding lifted vectors in $H_{q} Q \subset T_{q} Q$ under this identification. The horizontal lift, then, is the key to understanding the relationship between motion in the base space (via tangent vectors on $\left.T_{\pi_{2}(q)} M\right)$ and motion in the total space, $Q$, where locomotion is effected.

The connection is a general geometric structure that will enable us to describe how internal shape changes create net robot motion. This is the generalization and formalization of the intuitive procedure that lead to Eq. 5 . As shown in the snakeboard, the kinematic constraints, Eq. 2, are generally not sufficient to define the robot's motion. It is necessary to supplement the kinematic constraints with symmetry constraints, in the form of the constrained momenta, in order to define a connection. In order to do so, we must make two assumptions regarding $\mathcal{S}$. The first assumption, that the constraints be $G$-invariant, is a natural one when dealing with locomotion. Like the Lagrangian, locomotion constraints should not depend upon the robot's absolute position and orientation, and so are expected to be invariant with respect to the group. The second assumption is more technical in nature. Let $\operatorname{dim} G=s$. Then it is assumed that $\operatorname{dim} \mathcal{S}=\operatorname{dim} \mathcal{D}-\operatorname{dim} M=s-k$. Intuitively, this assumption implies that there are no constraints acting directly on the internal shape changes.

Given these assumptions, the constrained momenta described in Proposition 6 give the additional constraints necessary to construct a connection. Recall that Eq. 2 gives $k$ constraints, $\omega^{i}(q) \dot{q}=0, i=1, \ldots, k$, on an $s$ dimensional group. Given our dimensional assumption on $\mathcal{S}$, we can choose a basis for $\mathcal{S}$ and hence develop $s-k$ additional affine constraint equations, $\omega^{k+1}, \ldots, \omega^{s}$, from the constrained momenta,

$$
\omega_{j}^{k+i} \dot{q}^{j}=\left(p^{c}\right)^{i}=\frac{\partial L}{\partial \dot{q}^{j}}\left(\left(\xi^{c}\right)_{Q}^{k+i}\right)_{j}(q),
$$

where the $\left(\xi^{c}\right)_{Q}^{k+i}$ form a basis for $\mathcal{S}$, and hence each $\left(\xi^{c}\right)^{k+i}$ lies in $\mathfrak{g}$ (but may vary pointwise over $Q$ ). In order to establish an invariant horizontal distribution (i.e., satisfy condition (2) of Defn. 7), we must show that Eq. 14 is $G$-invariant.

Proposition 8. Given a system with $L$ and $\mathcal{D} G$ invariant, and for which there exists a $G$-invariant basis for $\mathcal{S}, X^{1}, \ldots, X^{s-k}$, the constrained momentum given by $\left(p^{c}\right)^{i}\left(v_{q}\right)=\left\langle\frac{\partial L}{\partial \dot{q}}\left(v_{q}\right) ; X^{i}\left(v_{q}\right)\right\rangle$ is itself $G$-invariant, i.e., $\left(p^{c}\right)^{i}\left(T_{q} \Phi_{h} v_{q}\right)=\left(p^{c}\right)^{i}\left(v_{q}\right)$, for $i=1, \ldots, s-k$.

The constrained momenta of Eq. 14 may then be appended to the kinematic constraints of Eq. 2 in order to define the fiber equations:

$$
\omega \dot{q}=\tilde{\gamma},
$$

where $\tilde{\gamma}=\left(0, \ldots, 0,\left(p^{c}\right)^{1}, \ldots,\left(p^{c}\right)^{s}\right)$ is an affine term stemming from the constrained momenta developed in Proposition 6. The motion of an undulatory system must satisfy the fiber equations.

Using the invariance of Eq. 15, we can separate the fiber equations into fiber and base components. First, rewrite Eq. 15 as

$$
\omega_{g}(g, r) \dot{g}+\omega_{r}(g, r) \dot{r}=\tilde{\gamma} .
$$

Invariance of Eq. 15 means $\omega_{g}\left(\Phi_{h} g, r\right) D_{q} \Phi_{h}=\omega_{g}(g, r)$ and $\omega_{r}\left(\Phi_{h} g, r\right)=\omega_{r}(g, r)$. Setting $h=g^{-1}$ in the former relation gives $\omega_{g}(g, r)=\omega_{g}(e, r) D_{q} \Phi_{g^{-1}}=\omega_{g}(r) g^{-1}$, while the latter relation implies that $\omega_{r}$ is independent of $g$. It can also be shown that $\omega_{g}(r)$ is invertible, and so Eq. 15 becomes

$$
g^{-1} \dot{g}=A(r) \dot{r}+\gamma(r) p^{c},
$$

where $g^{-1}$ represents the lifted action applied to vectors tangent to the fiber, and $\gamma(r) p^{c}=\omega_{g}^{-1}(r) \tilde{\gamma}$.

Practically speaking, the fiber equations, Eq. 15, play the most central role in the mechanics of undulatory locomotion. Formally, the connection is defined by setting $\tilde{\gamma}=0$ in Eq. 15. If $\tilde{\gamma}=\gamma=0$, then Eq. 17 describes the horizontal lift, i.e., the relationship between base vectors in $T_{\pi_{2}(q)} M$ and vectors in the full state space, $T_{q} Q$.

The relationship between the connection and the fiber equations can be interpreted as follows. Recall that $g^{-1} \dot{g}$ is an element of the Lie algebra, $\mathfrak{g}$, which physically corresponds to the velocity of the robot's body fixed reference frame (as seen by an observer in the body frame). The connection (described by $\tilde{\gamma}=\gamma=0$ in Eqs. 15, 17) directly describes how internal shape changing motions, $\dot{r}$, lead to robot motion, $g^{-1} \dot{g}$. However, the moving robot may have built up some momentum due to previous motions. When there is no motion in the base space $(\dot{r} \equiv 0)$, the robot's motion is driven solely by the momentum terms, $\gamma$. Thus, the fiber equations determine the robot's motion from the combination of built-up momentum and internal shape changes. They are formulated in a way 
which makes explicit each of these two contributions to locomotion.

However, the momentum terms in the fiber equations are themselves governed by the generalized momentum equation, Eq. 12. For the case of undulatory locomotion, where the constraints are assumed to be group invariant, it can further be shown that Eq. 12 is also invariant with respect to the group action.

Proposition 9. Given a constrained mechanical system, define the function $P^{c}(q, \dot{q})=\frac{\partial L}{\partial \dot{q}^{i}}\left(\frac{d}{d t}\left(\xi^{c}(q)\right)_{Q}^{i}\right)=$ $\frac{d}{d t} p^{c}$, where $\xi_{Q}^{c}$ is a $G$-invariant vector field in $\mathcal{S}$ and $\xi^{c}$ is the Lie algebra-valued function over $Q$ which generates $\xi_{Q}^{c}$. As a function on $T Q, P^{c}$ is $G$-invariant. Thus, for all $g \in G$,

$$
P^{c}\left(\Phi_{h} q, T_{q} \Phi_{h} \dot{q}\right)=P^{c}(q, \dot{q}) .
$$

Practically speaking, this proposition implies that Eq. 12 can always be expressed strictly in terms of base variables and constrained momenta, i.e.,

$$
\dot{p}^{c}=f\left(r, \dot{r}, p^{c}\right) .
$$

Example 2: (concl.) Combining the kinematic constraints and the constrained momentum, the fiber equations are:

$$
W(g, r) \dot{g}-\left(\begin{array}{c}
0 \\
0 \\
f(r) \dot{r}
\end{array}\right)=\left(\begin{array}{c}
0 \\
0 \\
p^{c}
\end{array}\right)
$$

where

$$
W(g, r)=\left(\begin{array}{ccc}
-\sin \left(\phi_{b}+\theta\right) & \cos \left(\phi_{b}+\theta\right) & -l \cos \phi_{b} \\
-\sin \left(\phi_{f}+\theta\right) & \cos \left(\phi_{f}+\theta\right) & l \cos \phi_{f} \\
m a & m b & \hat{J}_{c}
\end{array}\right),
$$

$$
f(r)=\sin \left(\phi_{b}-\phi_{f}\right)\left(J_{r}, J_{w}, J_{w}\right) .
$$

The invariance of the constraints and the constrained momentum allows us to extract the group variables from Eq. 19. Letting $e$ denote the identity group transformation, we have

$$
\begin{gathered}
W(e, r) g^{-1} \dot{g}= \\
\left(\begin{array}{ccc}
-\sin \phi_{b} & \cos \phi_{b} & -l \cos \phi_{b} \\
-\sin \phi_{f} & \cos \phi_{f} & l \cos \phi_{f} \\
-2 m l \cos \phi_{b} \cos \phi_{f} & -m l \sin \left(\phi_{b}+\phi_{f}\right) & \hat{J} \sin \left(\phi_{b}-\phi_{f}\right)
\end{array}\right) \\
\cdot\left(\begin{array}{ccc}
\cos \theta & \sin \theta & 0 \\
-\sin \theta & \cos \theta & 0 \\
0 & 0 & 1
\end{array}\right) \\
=\left(\begin{array}{c}
0 \\
0 \\
p^{c}-f(r) \dot{r}
\end{array}\right) .
\end{gathered}
$$

Finally, we can write this as

$$
g^{-1} \dot{g}=A(r) \dot{r}+\gamma
$$

where

$$
\begin{gathered}
A(r)=\frac{1}{\operatorname{det}[W(e, r)]} \cdot\left(\begin{array}{c}
2 l \cos \phi_{b} \cos \phi_{f} \\
l \sin \left(\phi_{b}+\phi_{f}\right) \\
-\sin \left(\phi_{b}-\phi_{f}\right)
\end{array}\right) \cdot f(r) \\
\gamma=\frac{p^{c}}{\operatorname{det}[W(e, r)]} \cdot\left(\begin{array}{c}
2 l \cos \phi_{b} \cos \phi_{f} \\
l \sin \left(\phi_{b}+\phi_{f}\right) \\
-\sin \left(\phi_{b}-\phi_{f}\right)
\end{array}\right) .
\end{gathered}
$$

The generalized momentum equation (Eq. 12) is

$$
\dot{p}^{c}=m \dot{a} \dot{x}+m \dot{b} \dot{y}+\hat{J} \dot{c} \theta+J_{r} \dot{c} \dot{\psi}+J_{w} \dot{c}\left(\dot{\phi}_{b}+\dot{\phi}_{f}\right) .
$$

Using the invariant form of the connection, it is possible to rewrite this equation solely in terms of the base variables and the momentum:

$$
\dot{p}^{c}=\frac{1}{2} \frac{d}{d t}\left(\log \left(-\operatorname{det} \omega_{g}(r)\right)\right) \cdot\left(p^{c}-f(r) \dot{r}\right)+\dot{f}(r) \dot{r},
$$

where $\omega_{g}(r)=\omega_{g}(e, r)$ as defined in Eq. 16, and $\operatorname{det} \omega_{g}(r)=-\left(m R^{2}+\hat{J}\right) c$ which corresponds to the inertia of the board about the instantaneous center of rotation (see Figure 3). Most importantly, however, notice that the dependence on the fiber variables has been completely eliminated from the generalized momentum equation.

\subsection{Summary and limiting cases}

In summary, by using a geometric approach, and by using the symmetries and constraints which are natural to undulatory locomotion systems, we have reduced the system of $n$ second order ODE's with $k$ first order constraints (Eqs. 2 and 3 ) to a system of $s$ first order (affine) constraints termed the fiber equations, $s-k$ first order generalized momentum equations (Eq. 18),

$$
\begin{aligned}
g^{-1} \dot{g} & =A(r) \dot{r}+\gamma(r) p^{c} \\
\dot{p}^{c} & =f\left(r, \dot{r}, p^{c}\right)
\end{aligned}
$$

and a group of second order equations on the base space. This paper does not discuss the "reduced dynamics" on the base space, as these dynamics are not as important for the understanding of undulatory locomotion. Thus, the equations which are important to understanding undulatory locomotion are reduced to two first order equations that make explicit how internal shape changes and physical inertial lead to robot motion. We now briefly consider special cases of these equations that have occurred in previous work. For additional discussion of these ideas, the reader is referred to [3], [7].

Purely kinematic constraints. With a sufficient number of kinematic constraints, the system's motion along the fiber is fully constrained. In this kinematic case, the connection takes the simpler form:

$$
g^{-1} \dot{g}=A(r) \dot{r} \text {. }
$$

Most commonly studied wheeled vehicles, such as Example 1, fall into this category. Eq. 22 describes a system on the fiber with no drift - a case that has been extensively studied in the literature on nonholonomic systems.

Pure symmetry constraints. There are no kinematic constraints in the cases of falling cats, satellites 
with rotors or attached robot arms, and platform divers. However, all of these systems have inherent Lagrangian symmetries, and therefore conserved momenta. In these cases, the fiber and generalized momentum equations take the form:

$$
\begin{aligned}
g^{-1} \dot{g} & =A(r) \dot{r}+\beta(g, r) \mu \\
\dot{\mu} & =0 .
\end{aligned}
$$

$\mu$ is the generalized momenta, and is constant. If $\mu=0$, these equations reduce to Eq. 22. Using $A(r)$ it is clear how internal shape changes lead to net reorientation of the system. The use of fiber bundles and connections plays a primary role in understanding the mechanics and control of systems with dynamic constraints.

\section{Discussion}

Undulatory locomotors have no jets, thrusters, tracks, or legs to generate motion. Instead, motion is generated by a coupling of internal shape changes to external constraints. This paper has focused on systems with nonholonomic kinematic constraints. This class of systems includes not only the snakeboard, but also the "active cord" mechanism of Hirose [5], and any terrestrial undulatory robotic system that uses wheels to provide motion constraints. Also, many of the snake and worm-like systems discussed in [4] can be analyzed using the techniques described here. Fish and some other undulatory mechanisms seem to use a similar principle to generate movement, but the constraints are more complicated than the ones considered in this paper. It is possible that our framework can be extended to include these systems as well. Furthermore, the same basic process seems to exist in legged locomotion, though the discontinuous nature of the dynamics makes it more difficult to analyze these types of systems from a classical standpoint.

A key observation in this work is that the constraints inherent in undulatory systems provide the means to determine motion as a function of internal shape change. When kinematic constraints are not sufficient to uniquely determine the robot's motion, dynamic symmetries provide the additional constraints. of connections on principle bundles to describe the relationship between internal deformations and locomotive effect because the connection encompasses much of the information which is essential to locomotion. Using these tools, we can parameterize the dynamics in terms of physically meaningful variables of generalized momenta, internal shape, and motion of the robot reference frame.

While this paper introduced a useful framework for studying undulatory mechanics, many open questions remain. Controllability of undulatory systems is a central issue that remains unresolved. Given an initial configuration, $q_{0}$, and a final configuration, $q_{1}$, an undulatory robot such as the snakeboard is said to be controllable if there exists a path connecting $q_{0}$ to $q_{1}$ which satisfies the robot's dynamical equations. Using a principle fiber bundle framework, Kelly and Murray [7] have recently derived a controllability test for systems which are described by the kinematic connection of Eq. 22. We believe that the formulation developed in this work will ultimately lead to an analogous controllability test for more general classes of systems which include dynamic constraints. Beyond the question of controllability lies the practical importance of developing motion planning schemes to generate feasible, or perhaps optimal, paths for undulatory motion planning problems. Finally, we wish to develop a better understanding of the concept of a "gait" in undulatory systems. For example, in [11] it was shown that the snakeboard exhibits different "gaits" which generate motion. The geometric interpretation of these gaits is still unclear.

\section{References}

[1] R. Abraham and J. Marsden. Foundations of Mechanics. Addison-Wesley, Reading, MA, second edition, 1978.

[2] M. Berkemeier and R. Fearing. Control of a two-link robot to achieve sliding and hopping gaits. In Proc. IEEE Int. Conf. on Robotics and Automation, pages 286-291, Nice, France, May 1992.

[3] A. M. Bloch, P. S. Krishnaprasad, J. E. Marsden, and R. M. Murray. Nonholonomic mechanical systems and symmetry. Available electronically via http://avalon.caltech.edu /cds/reports/cds94-013.ps, California Institute of Technology, Pasadena, CA, 1994.

[4] G. S. Chirikjian and J. W. Burdick. Kinematics of hyperredundant locomotion with applications to grasping. In Proc. IEEE Int. Conf. on Robotics and Automation, pages 720-727, Sacramento, CA, April 1991.

[5] S. Hirose and Y. Umetani. Kinematic control of active cord mechanism with tactile sensors. In Proc. $2^{\text {nd }}$ Int. CISM-IFT Symp. on Theory and Practice of Robots and Manipulators, pages $241-252,1976$.

[6] S. Kajita and K. Tani. Study of dynamic biped locomotion on rugged terrain. In Proc. IEEE Int. Conf. Robotics and Automation, pages 1405-1411, Sacramento, CA, April 1991.

[7] S. D. Kelly and R. M. Murray. Geometric phases and locomotion. Available electronically via http://avalon.caltech.edu /cds/reports/cds94-014.ps. To appear, J. Robotic Sys., CIT, Pasadena, CA, 1994.

[8] S. Kobayashi and K. Nomizu. Foundations of Differential Geometry, volume I. John Wiley and Sons, New York, 1963.

[9] D. Koditschek and M. Bühler. Analysis of a simplified hopping robot. Int. J. Robotics Research, 10(6):587-605, 1991.

[10] J. Koiller. Reduction of some classical non-holonomic systems with symmetry. Archives for Rational Mechanics and Analysis, 118(2):113-148, 1992 .

[11] A. D. Lewis, J. P. Ostrowski, R. M. Murray, and J. W. Burdick. Nonholonomic mechanics and locomotion: the snakeboard example. In Proc. IEEE Int. Conf. Robotics and Automation, pages 2391-2397, San Diego, CA, May 1994.

[12] T. McGeer. Passive dynamic walking. Int. J. of Robotics Research, 9(2):62-82, Apr 1990.

[13] J. P. Ostrowski, A. D. Lewis, R. M. Murray, and J. W. Burdick. Reduction of mechanical systems with constraints. In preparation, 1995.

[14] M. H. Raibert. Legged Robots that Balance. MIT Press, Cambridge, MA, 1986.

[15] A. Shapere and F. Wilczek. Geometry of self-propulsion at low Reynolds number. J. Fluid Mech., 198:557-585, 1989.

[16] S. Song and K. Waldron. Machines that Walk: the Adaptive Suspension Vehicle. MIT Press, Cambridge, MA, 1989. 